\title{
Implementing and evaluating standardised tuberculosis incident management for nonhousehold contacts in a large clinical network
}

\begin{abstract}
To the Editor:
Copyright @The authors 2021

This version is distributed under the terms of the Creative Commons Attribution NonCommercial Licence 4.0. For commercial reproduction rights and permissions contact permissions@ersnet.org

Received: 19 April 2021 Accepted: 27 May 2021

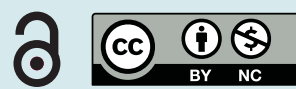

The strategy to eliminate tuberculosis (TB) in low-incidence countries includes the investigation of the contacts of TB cases [1]. This recognises that whilst most TB disease in these settings is due to reactivation [2], local transmission also occurs [3]. In the UK, TB networks (TBN), which support and coordinate local and regional TB services, typically follow the National Institute of Health and Care Excellence guidance which recommends screening close contacts (predominantly household) but not routinely for social or non-household contacts [4]. Many studies from low TB burden countries report contact investigation for specific congregate settings including outbreaks in childcare centres [5], homeless facilities [6] and methadone treatment clinics [7]. However, the comprehensive application of contact investigation across multiple non-household locations is rarely reported. Therefore, we evaluated a systematic approach to managing people potentially exposed to TB in congregate settings.

We identified all potential TB transmission incidents occurring in a non-household setting and reported within a London TBN between 1 June 2016 and 31 December 2018. The North-Central London TBN covers four TB services that are responsible for a population of 1.6 million residents. It treats 250-300 cases of TB disease each year. Non-household TB incidents are managed within a pathway involving referral by the clinical TB service to the local Public Health England (PHE) Health Protection Team (HPT) who have a legal duty to maintain public standards of infection prevention and control. Detailed information is included in the referral to facilitate a risk assessment conducted in accordance with agreed PHE procedures (unpublished data). The process is led by a dedicated TB nurse working with TB services, local authorities and the HPT. The nurse also provides prevention-education messages appropriate to the incident.

Incidents were defined as: 1) contact $>8 \mathrm{~h}$ per week with an infectious TB case in an indoor congregate setting; or 2) contact with immuno-compromised individuals in that setting of any duration.

Data were collected on the incident, the contacts identified, and the number screened for latent TB infection (LTBI), or who were sent written information regarding their probable low risk of TB following the exposure.

Demographic and clinical characteristics of the index cases were extracted from the London TB notification register. These included factors considered to be associated with infectiousness, such as sputum acid fast bacilli smear positivity and cavitation on chest radiographic imaging.

The LTBI yield from screening and the treatment decisions were compared with household contacts screened contemporaneously by the TBN, using Chi-squared testing. LTBI yield stratified by presumed index case infectiousness was assessed using the Kruskal-Wallis test. LTBI treatment rate and the number needed to screen to detect one LTBI case were compared between household and congregate settings using Chi-squared testing.

Shareable abstract (@ERSpublications)
A systematic approach to nonhousehold TB contact identified a similar number of LTBI cases to
household screening over the same time period https://bit.ly/2Tq96LN

Cite this article as: Patterson B, Boparai N, Dekoningh J, et al. Implementing and evaluating standardised tuberculosis incident management for nonhousehold contacts in a large clinical network. ERJ Open Res 2021; 7: 00270-2021 [DOI: 10.1183/23120541.00270-2021].
\end{abstract}


The HPT and nurse-led TBN screening team were referred, and systematically evaluated 202 potential incidents. These were reported from 86 (42.6\% of total) healthcare and 116 (57.4\%) community settings. 57 (66.3\%) in healthcare were from hospital inpatient areas, 23 (26.7\%) from emergency departments, five (5.8\%) from nursing homes and one (1.2\%) from a primary care centre. In the community, 28 (24.1\%) were from educational institutions, 18 (15.5\%) workplaces/offices, six (5.2\%) hostels/homeless shelters, $11(9.5 \%)$ related to airline travel (which were not further assessed by our service) and 53 (45.7\%) from other venues including pubs, restaurants, religious centres and shops.

Following the risk assessment, 101 (50.0\%) possible incidents required further investigation. These were most often in healthcare settings, followed by other venues (figure 1). The 101 incidents were associated with 82 index cases, 63 of whom resulted in one incident and 19 with two incidents. 4038 potential non-household contacts were identified, and 2455 (60.8\%) were screened. A further 1399 (34.6\%) were sent "inform and advise" letters, 19 died before assessment, 34 were referred to external clinics for management, and 152 were lost to follow-up.

A median (interquartile range) of 11.5 (7-24) contacts were screened per incident. 44 (43.6\%) out of 101 investigated incidents, representing $39(47.6 \%)$ of the index cases, yielded no LTBI cases. These were most often seen in healthcare and other venues settings (figure 1). Overall, 165 (6.7\% of those screened) LTBI and two (0.08\%) TB cases were identified (figure 1). 105 (63.6\%) LTBI cases started treatment.

Over the same time interval 1583 household contacts were screened in our network, 155 (9.8\%) were diagnosed with LTBI, and 13 (0.8\%) with TB disease. 148 (95.5\%) LTBI cases started treatment ( $\mathrm{p}=0.02$ compared to frequency of starting treatment in non-household contacts). The mean number needed to screen to identify a case of LTBI was 10.2 for household contacts compared with 17.0 for non-household contacts $(\mathrm{p}<0.00001)$. TB disease was detected at a 10-fold higher rate in household contacts.

There were no significant differences in the yield of LTBI cases after stratification by sputum smear status or presence of cavitation on chest radiography $(\mathrm{p}=0.46)$.

A systematic approach to non-household TB contact assessments has rarely been reported. This study has identified a large number of incidents requiring input from TB and public health services. We found that in a metropolitan TBN, non-household contact tracing identified a similar number of LTBI cases to household screening over the same time period. However, this involved assessing more non-household contacts and therefore had a considerably lower yield for LTBI detection. In addition, fewer TB cases were identified.

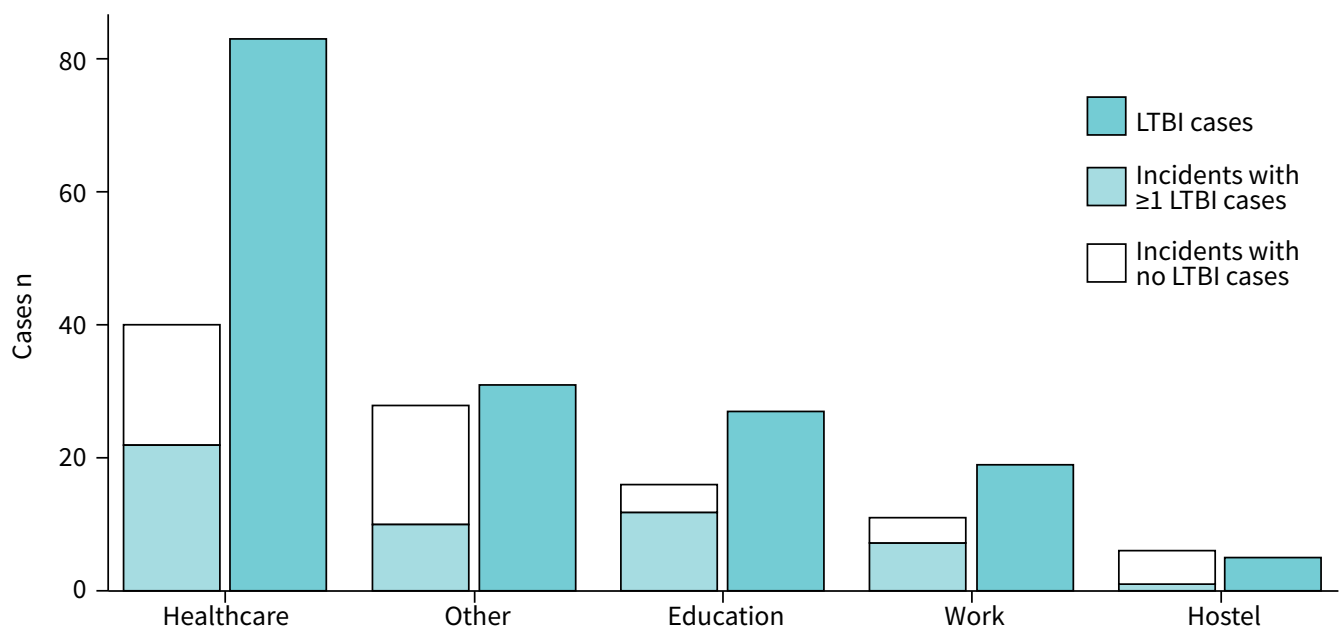

FIGURE 1 Number of incidents associated with each setting where screening was required, divided into those with at least one associated case of latent tuberculosis infection (LTBI) and those with no associated cases of LTBI. The total number of associated LTBI cases identified following screening for each setting are also shown. Other settings comprise pubs, religious centers, restaurants, shops etc. 
A key aim of contact tracing is to find infected contacts and offer them treatment. While we observed high treatment uptake in household contacts (96\%), non-household contacts were less likely to start treatment for LTBI (67\%).

Of note, in almost $50 \%$ of reported possible incidents further assessment was not required, suggesting that the criteria used to identify these events could be improved. As nearly half the incidents and index cases investigated were not responsible for any onward infections, there is further scope to refine the current approach and target those incidents most likely to result in LTBI and TB disease. However, stratification by index case smear-positivity or presence of cavitary disease (clinical features that might be expected to be associated with infection transmission) did not increase the LTBI yield.

Healthcare settings (the commonest site of incidents) had the highest proportion where no LTBI cases were identified. Thus, whilst specific incident details such as duration of exposure to the index case, room size and ventilation may help identify where screening will provide greatest yield, an alternative approach would be to develop a more robust assessment for hospital incidents that would reduce the numbers requiring full contact tracing. This, however, may not be straightforward given the reputational issues that can be associated with an incident of possible TB transmission in a hospital setting.

We believe our model offers a simple and effective approach to TB control and management of non-household TB incidents. Our dedicated TBN screening lead provided a systematic method of assessing non-household contacts to facilitate standardised care, better data, and quality improvement across our network. Our approach offers the added value of enabling targeted education on TB transmission and infection control to be provided to people in "hot-spot" incident settings such as healthcare environments, schools, colleges and places of work.

Benjamin Patterson $\oplus^{1}$, Narinder Boparai ${ }^{2}$, Jennifer Dekoningh ${ }^{2}$, Sudy Anaraki ${ }^{3}$, Jacqui White ${ }^{2}$ and Marc Lipman $\mathbb{0}^{1,4}$, on behalf of the North Central London TB Network

${ }^{1}$ Royal Free London NHS Foundation Trust, London, UK. ${ }^{2} \mathrm{NCL}$ TB Service, Whittington Health, London, UK. ${ }^{3}$ North East and North Central London Health Protection Team, Public Health England, London, UK. ${ }^{4}$ UCL Respiratory, University College London, London, UK.

Corresponding author: Benjamin Patterson (patterson.b@unic.ac.cy)

Conflict of interest: The authors declare no competing interests.

References

1 Lönnroth K, Migliori GB, Abubakar I, et al. Towards tuberculosis elimination: an action framework for low-incidence countries. Eur Respir J 2015; 45: 928-952.

2 Shea KM, Kammerer JS, Winston CA, et al. Estimated rate of reactivation of latent tuberculosis infection in the United States, overall and by population subgroup. Am J Epidemiol 2014; 179: 216-225.

3 Cavany SM, Vynnycky E, Sumner T, et al. Transmission events revealed in tuberculosis contact investigations in London. Sci Rep 2018; 8: 6676.

4 NICE National Institute for Health and Care Excellence. Tuberculosis. NICE guideline [NG33]. www.nice.org.uk/ guidance/ng33 Date last updated: 12 September 2019. Date last accessed: 1 March 2021.

5 Gaensbauer JT, Vandaleur M, O'Neil M, et al. BCG protects toddlers during a tuberculosis outbreak. Arch Dis Child 2009; 94: 392-393.

6 Lofy KH, McElroy PD, Lake L, et al. Outbreak of tuberculosis in a homeless population involving multiple sites of transmission. Int J Tuberc Lung Dis 2006; 10: 683-689.

7 Conover C, Ridzon R, Valway S, et al. Outbreak of multidrug-resistant tuberculosis at a methadone treatment program. Int J Tuberc Lung Dis 2001; 5: 59-64. 